\title{
Evaluation of Treatment of Degenerative Lumbar Disorders by Posterior Interbody Fusion Using Single Cage
}

\author{
Authors \\ Walid El Nawawy, Mohamed Aly, Ali Ibrahim Ahmed, Ahmed M. Bassiony Ismail, \\ Bishoy Bessada, Amr Eltorky, Ramy Shehata, Mohamed S. A. A. Hamed, \\ Alaa El Banna, Aly Salama \\ Department of Orthopedics Surgery and Traumatology, Faculty of Medicine, University of Alexandria
}

\begin{abstract}
Background: Spinal stenosis is defined as narrowing in the spinal canal or neural foramina, and this can cause compression on the spinal cord, cauda equina or individual nerve roots. It may be congenital or acquired. The acquired one is much more common and can result from facet osteoarthritis, ligamentum flavum hypertrophy, degenerative bulging disc or osteophyte formation.

Studies show that conservative management might be successful in 70-90\% of patients.

Aim: The aim of this work was to assess the early results of posterior lumbar interbody fusion (PLIF) using single TLIF PEEK cage in the treatment of lumbar degenerative disorders.

Patients and Methods: This prospective study included 20 patients with lumber disc prolapse or lumbar spinal stenosis treated surgically with posterior lumber interbody fusion (PLIF) using single PEEK TLIF cage. Instability, post-laminectomy syndrome and cases with haematogenous infection were excluded from this study. All surgeries were performed at El-Hadara University Hospital, Alex, Egypt. Patients were followed up at for at least 6 months. Informed consent were taken from all patients. A single TLIF cage filled with local bone graft was inserted. Visual analogue scale (VAS) was used for back and leg pain.

Results: At the end of the follow up period, 8 patients (40\%) had excellent results, resuming unrestricted activity, near complete relief of pain in the back, lower limbs or both. Ten patients (50\%) had good results, resuming unrestricted activity, significant improvement in pain with only occasional discomfort in the back or lower limbs, necessitating non-narcotic medication. Two patients (10\%) had fair results showing restriction of activities, some improvement but still had intermittent discomfort in the back and lower limbs, needing sometimes non-narcotic medication.
\end{abstract}

Conclusion: All cases after PLIF using single PEEK cage showed adequate fusion.

\section{Introduction}

Lumbar disc prolapse and lumbar canal stenosis are common sequale of degenerative disorders of the spine. $^{(1)}$

Spinal stenosis is defined as narrowing in the spinal canal or neural foramina, and this can cause compression on the spinal cord, cauda equina or individual nerve roots. It may be congenital or acquired. The acquired one is much more common and can result from facet osteoarthritis, ligamentum flavum hypertrophy, degenerative bulging disc or osteophyte formation. ${ }^{(2)}$

Conservative therapy in the form of life style modification, reassurance and adequate analgesia that 
can help a significant number of patients. If the patient's quality of life is deteriorating and the MRI findings match the symptoms and signs, surgery can be considered. ${ }^{(2)}$

Lumbar disc prolapse (LDP) may be protrusion, extrusion or sequestration. $^{(3)}$

LDP can present as low back pain which is exacerbated by certain activities, with or without radiculopathy. ${ }^{(4)}$

Ninety percent of LDP can be treated efficiently with conservative treatment, ${ }^{(5)}$ which is indicated in young age, minor neural compromise, small herniation, mild disc degeneration, and mild to moderate sciatica. ${ }^{(6)}$

Surgical indications in the treatment of LDP are either absolute indications (cauda equina syndrome, severe paresis and paraparesis), or relative indications (severe sciatica, persistent radicular pain not responding to treatment, persistent sensorimotor deficit). ${ }^{(7)}$

Surgical treatment for LDP and lumbar spinal stenosis can be in the form of decompression with or without fusion. Fusion can be posterolateral or interbody fusion. The later can be achieved through different approaches as anterior lumbar interbody fusion (ALIF), transforaminal lumbar interbody fusion (TLIF) and posterior lumbar interbody fusion (PLIF). The justification for interbody fusion over posterolateral fusion is that placing bone graft or cage in the anterior and middle spinal columns will increase the chance for bony fusion. ${ }^{(8)}$

Several types of cages are available as metal, carbon fiber, polymethylmethacrylate (PMMA) and polyetheretherketone (PEEK). ${ }^{(8)}$

PLIF cages can be used either single or double. There are several practical problems encountered in the procedure with implantation of two cages, such as difficulty in achieving symmetric positioning with two cages, loosening of the first cage following insertion of the second one, retropulsion or displacement of the cages with or without nonunion. $^{(9)}$

Some surgeons prefer to use a single cage in order to avoid such complications.

\section{Patients and Methods}

This study included 20 adult patients suffering from symptomatic degenerative lumbar disc prolapse and /or lumbar canal stenosis of varying grades.

These patients were considered and indicated for surgical treatment due to failure of response to or relief of symptoms by conservative treatment for more than 3 months, or they presented initially with neurological deficits.

All patients in this study were treated by posterior lumbar interbody fusion using cages supplemented by transpedicular screw fixation.

All patients were thoroughly informed about the procedure, its benefits and risks, approximate recovery time postoperatively and possible complications. An informed consent was taken from every patient.

\section{Analysis of Patients}

\section{1- Age}

Patients' ages ranged from a minimum of 24 years old to a maximum of 65 years old with a mean age of $45.35 \pm 10.5$ years old

\section{2- Sex}

Out of the 20 patients of this study, there were 14 female patients $(70 \%)$ and six male patients $(30 \%)$

\section{Results}

At the end of the follow up period, 8 patients (40\%) had excellent results, resuming unrestricted activity, near complete relief of pain in the back, lower limbs or both. Ten patients $(50 \%)$ had good results, resuming unrestricted activity, significant improvement in pain with only occasional discomfort in the back or lower limbs, necessitating non-narcotic medication. Two patients $(10 \%)$ had fair results showing restriction of activities, some improvement but still had intermittent discomfort in the back and lower limbs, needing sometimes non-narcotic medication. 


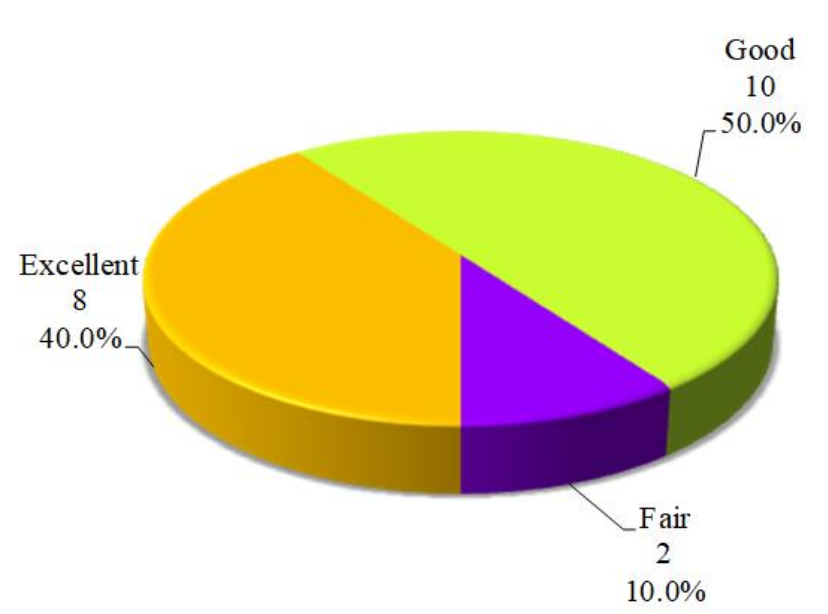

When comparing our pre-operative and postoperative clinical assessments of the 20 patients in this study, we found that out of the eight patients rated as excellent postoperatively, one was rated as good, five were rated as fair and two patients were rated as poor pre-operatively. Out of the ten patients rated as good post-operatively, seven were rated as fair, one was rated as good and two were rated as poor pre-operatively. The results showed that the two patients who were rated as fair post-operatively were also rated as fair preoperatively. The results were found to be statistically significant.

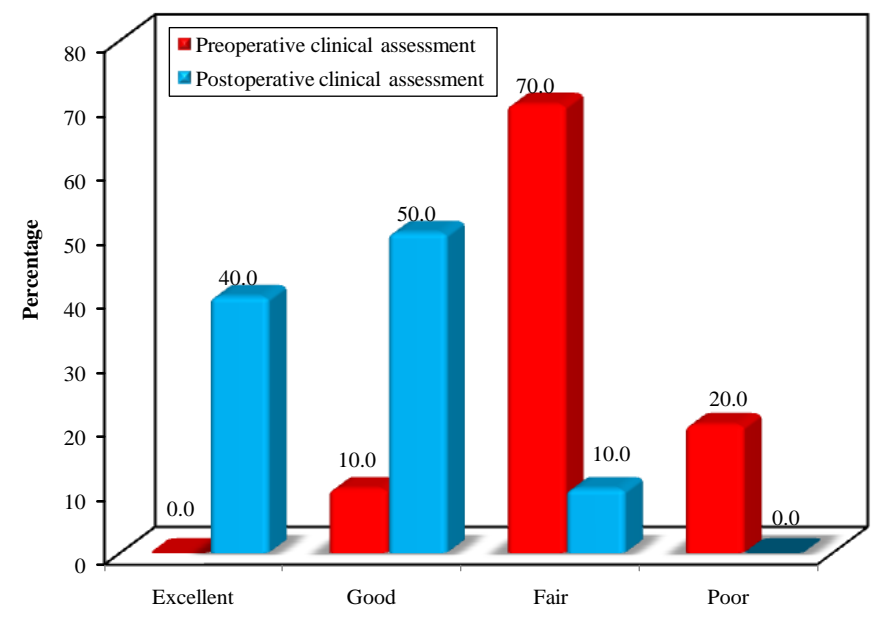

\section{Discussion}

Epidemiologic studies show that by the age of 50 years, $50 \%$ of the population experience LBP. By the age of 60 , the cumulative incidence is over $80 \%$. It is present in all societies and cultures, although it may be experienced differently. ${ }^{(10)}$
Acute LBP is defined as LBP lasting less than 3 months.

LBP lasting more than 3 months is defined as chronic LBP. Recent studies report that a significant percentage of patients who present with acute LBP continue to experience recurrent or persistent symptoms. ${ }^{(11)}$

Most degenerative spinal conditions should be treated conservatively first. Surgery is obviously elective and can be done when the patient is medically fit. ${ }^{(12)}$

Although the primary goal is to relieve the patient's leg symptoms, one must not lose sight of the mechanical back pain that is present. It is important to explain to patients before surgical intervention that although relief of leg pain often occurs, it may not be complete and some back pain will almost certainly remain. But the residual symptoms should not restrict activities nearly to the extent of the preoperative symptoms.

Two surgical procedures, alone or in combination, are performed: (a) decompression of neurologic structures and (b) stabilization of vertebral elements (fusion). ${ }^{(13)}$

However, there is still a debate about which fusion method is optimal. Theoretically, interbody fusion cages are useful to increase and maintain neuroforaminal height and to improve the chances of achieving a successful fusion. ${ }^{(14)}$

The literature could not prove so far a significant difference between PLIF procedures and instrumented or non-instrumented posterolateral fusion (PLF) in respect to clinical outcome or fusion rate. PLIF ensured a better sagittal balance, but is associated with a possible higher complication rate The PLIF procedure is a surgically demanding procedure that should only be performed by experienced spine surgeons as otherwise it can result in a far longer operating time, more blood loss, a higher complication rate, and most importantly neurological deterioration. ${ }^{(15-18)}$

In this study, it was assumed that solid fusion would provide good clinical outcomes, patients were assessed clinically and radiologically before, 
after the procedure and following the procedure by a period of 6 to 24 months to compare the results and determine the success rate of PLIF with regards to clinical outcome supported by radiological evidence of fusion.

However, this study has its limitations. Firstly, the assessment of fusion status was not optimally objective, especially with implants in the grafted sites. Secondly, there was still concern regarding the heterogeneity between patients with lumbar disc prolapse and lumbar canal stenosis. Although these two disease groups have a different pathogenesis, it is believed that treatment principles are the same. Thus, it was thought it was reasonable to put them together in this study.

Thirdly, the follow up period of 6 months was rather insufficient, and this may have influenced the outcome assessment owing to possible adjacent segment disease seen with longer follow up periods. Yet this problem was inherent in this study design and it was not possible to solve it in our study.

Finally, this study was conducted on 20 patients only, this yielded less than optimal results when considering the accuracy of the information, that is to say that each single patient represents $5 \%$ of the results. Therefore, the information gained would not be as accurate or refined as a study done on a larger number of patients, yet this problem too, was inherent to this study design and it was not possible to solve it in this study.

The overall pre-operative clinical assessment; initially 14 of the patients (70\%) showed restriction in their daily activities due to pain and were rated as fair, while 4 of the patients (20\%) showed a significant restriction in activity with severe back pain and were rated poor. However, 2 of the patients $(10 \%)$ had minimal restriction of activity and showed a good clinical assessment but were included in our study as they still had low back pain and sciatica not responding to adequate conservative treatment.

At the end of the follow up period; As regards to Sciatica, 18 patients $(90 \%)$ have shown immediate postoperative improvement while two patients $(10 \%)$ had not improved by the end of the follow up period. This may be attributed to the long duration of symptoms before the surgery as these patients had their symptoms for more than 2 years, this long duration lead to long standing nerve root compression resulting in nerve root fibrosis

As for Neurogenic claudication; post-operatively five out of the six patients $(83.33 \%)$ have improved, while one patient (16.67\%) showed no improvement. This may be attributed to the duration of symptoms before the surgery as the patient who didn't improve had his symptoms for more than a year and less than 2 years or may be due to inadequate decompression.

With regards to neurological deficit, three out of four patients $(75 \%)$ with sensory deficits have improved completely, while one patient $(25 \%)$ showed no post -operative sensory improvement at the end of the follow up period. This may be attributed to the long duration of symptoms before the surgery as the patient who didn't improve had his symptoms for more than 2 years. Patients with partial foot drop recovered with full power regained at the end of the follow up period.

The overall post-operative clinical assessment showed that; eight patients (40\%) had excellent results, 10 patients $(50 \%)$ had good results and two $(10 \%)$ had fair results showing no statistical significance between the L4-5 and L5-S1 level groups.

In a study of 20 patients treated by PLIF using the Harms cage and posterior fixation, Allam ${ }^{(19)}$ stated that the clinical improvement was rated as excellent in 16 cases (80\%), good in two patients $(10 \%)$ and two patients $(10 \%)$ were fair. The patient who showed non-union had a clinical rating as excellent.

In another study by Yan et al, ${ }^{(20)}$ there were 42 cases $(49.4 \%)$ rated excellent, $29(34 \%)$ rated good, $11(12.9 \%)$ rated fair, and three (3.5\%) rated poor in that study.

In a prospective study by Sears, ${ }^{(21)}$ on 34 cases with lumbar degenerative disorder, using titanium, 
carbon and PEEK cages he reported $91 \%$ satisfactory clinical outcome.

In a retrospective case study of the use of posterior lumbar interbody fusion (PLIF) to treat lumbar foraminal stenosis (LFS), data from 31 patients from 2001 to 2005 were analyzed. The affected levels were: L4/5 in 13, and L5/S1 in 18. The Japanese Orthopedic Association score improved from 17.1 points preoperatively to 24.9 points in the L4-5 group and from 15.5 points preoperatively to 21.2 points postoperatively in the L5-S1 group at final follow-up, and the overall fusion rate was $100 \%$ in both groups. Also, there was no difference in the complication rates for both groups. ${ }^{(22)}$

In this study, with regards to level of pain in the lower limb initially; the mean pre-operative level of pain in the lower limb was $6.95 \pm 1.35$, while the mean post-operative level of pain in the lower limb at the end of the follow up period was

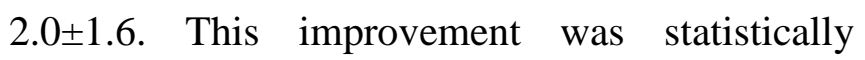
significant $(\mathrm{P}<0.0001)$.

As regards to level of pain in the back initially; the mean pre-operative level of pain in the back was $6.75 \pm 1.25$, while the mean post-operative level of pain in the back at the end of follow up was $3.50 \pm 1.43$. This difference was statistically significant $(\mathrm{P}<0.0001)$. These results show a significant improvement in the patients' level of pain in the back.

As regards to level of activity; the mean preoperative level of activity was $5.65 \pm 0.98$, while the mean post-operative level of activity was $8.25 \pm 1.01$. This difference was statistically significant $(\mathrm{P}<0.0001)$, showing a significant improvement in the level of activity in these patients. Our results were comparable to the study done by Yan et al. ${ }^{(23)}$

When comparing between the VAS scale for level of activity of the patients pre and postoperatively at the end of the follow up, it was found that the mean change of the VAS score for patients who underwent surgery after less than 1 year was $176.39 \pm 99.17$, while those who underwent surgery after more than a year and less than 2 years had a mean of $83.33 \pm 43.03$ and those who had their surgery after 2 years had a mean of $35.65 \pm 27.42$. This difference between the three groups was statistically significant $(\mathrm{P}<0.0001)$. These results showed a significant improvement in the level of activity of patients who had their surgery in less than a year from the start of their symptoms.

As for the radiological results of our study, we had a fusion rate of $100 \%$ which is comparable to most studies, Cloward ${ }^{(24)}$ had a $92 \%$ fusion rate in all cases of PLIF that he had done. Yan et al showed a fusion rate of $100 \%$, Allam stated that, 19 patients $(95 \%)$ had shown fusion at the end of follow up.

In a more recent study by $\mathrm{Yu}$ et $\mathrm{al},{ }^{(25)}$ they compared fusion rates of PLIF using bone chips only in 34 patients, titanium cages in 31 cases and PEEK cages in 11 patients. They showed fusion rates of $88.24 \%$ in patients with bone grafts only, $93.55 \%$ when using titanium cages and $100 \%$ with PEEK cages.

In the series of Csecsei et al ${ }^{(26)}$, they reported a 95.7\% fusion rate in 46 patients with the use of posterior elements taken from the decompression procedure as bone grafts. La Rosa et al (27), reported a $100 \%$ fusion rate in 17 cases using a titanium cage and autogenous iliac crest (AIC) bone graft. Chen et al ${ }^{(26)}$, in a study done on 118 patients, reported a $95 \%$ fusion rate using a Bagby and kuslich (BAK) cage filled with local bone chips. Furthermore, Zhao et al ${ }^{(28)}$, reported $100 \%$ fusion rate in 27 patients using BAK cages and AIC bone graft. While Kai et al ${ }^{(29)}$, reported only $92.9 \%$ fusion rate in a study done on 42 patients using local bone graft.

In this study we used cage and local bone graft with a fusion rate of $100 \%$. This may point to the good result of local bone to the morbidities of donor site and to the decrease of blood loss.

In this study, as regards to complications, we had one case $(5 \%)$ of superficial wound infection that responded well to treatment by oral antibiotics and daily dressing with topical disinfectant (betadine). One of our patients (5\%) had intra- 
operative dural tear which was managed intraoperative and showed clinical improvement with follow up. We had no cases of cage retropulsion or displacement, deep infection or implant failure such as screw breakage or coupling failure.

In the study by Yan et ${ }^{\mathrm{al}(24)}$, they reported that there were four complications, including three cases of radiculitis and one case of screw loosening. Allam ${ }^{(12)}$ reported one case $(5 \%)$ of screw breakage, however he reported full fusion without further surgical intervention. Allam also reported 3 cases $(15 \%)$ of cage malposition, one patient showed upward displacement of the cage and resulted in non-union at the end of follow up, while the remaining two patients showed cage rotation but fusion was nevertheless achieved for both of them at the end of the follow up period.

$\mathrm{Yu}$ et $\mathrm{ll}^{(30)}$ reported two cases of implant breakage in the cases where they did not insert a cage. They also stipulate that this might have been the result of a weaker mechanical spinal construct using unstructured bone grafts (without cages).Yu et al also stated that they found that artificial cages provided better functional and radiographic outcomes than only bone chips in PLIF, whereas both techniques(with and without cages) achieved comparable fusion rates. Their data also suggested that clinical outcomes correlate with radiographic fusion.

Ames et al found no significant difference in flexibility across grafted levels for any motion (flexion-extension, lateral bending, or axial rotation) when comparing an intact specimen with a single-level PLIF. The addition of pedicle screws after single-level interbody graft placement did, however, increase rigidity and subsequently decreased graft dislodgement and/or loosening. This modest improvement of stability for a single-level fusion was found to be drastically enhanced for a two-level fusion with the likely clinical correlation of a lower pseudoarthrosis rate. ${ }^{(19)}$

Ames et $\mathrm{al}^{(21)}$, found that after a two-level instrumentation procedure, the PLIF had a lower stabilizing effect on flexion-extension movement across the operated level than other techniques. The most likely cause of the relative functional instability of the PLIF procedure when performed over two levels is the more extensive facet and disc removal. Violation of the bilateral facet joints and anterior disc spaces across two levels after the PLIF technique seems to necessitate additional posterior column support to maintain sagittal plane balance.

Regarding the use of a single cage versus 2 cages, in a level III-2 retrospective study of 26 consecutive patients treated with a unilateral cage asking whether fusion healing and clinical outcome is comparable with that obtained with bilateral cages, there were no pseudoarthroses, instrumentation failures, or significant subsidence at any of the single cage levels. Disc space height and foraminal height were restored by the surgery and maintained at last follow-up. ${ }^{(23)}$

In another study, Follow up of 46 patients with degenerative lumbar spinal disease underwent single-level instrumented PLIF surgery using single closed box titanium cage for a mean period of 8 years showed similar radiologic results compared to fusion with 2 titanium cages and concluded that implantation of a single titanium closed-box cage in an instrumented PLIF seemed to be adequate in case of degenerative lumbar spinal disease. ${ }^{(30)}$

\section{References}

1.Buchowski JM. The Thoracic and Lumbar Degenerative Spine. In: Bridwell $\mathrm{KH}$, Dewald RL, editors. The Textbook of Spinal Surgery. $3^{\text {rd }}$ ed. Philadelphia, Lippincott Williams \& Wilkins 2011; 1(37):353.

2.Skyrme AD, Selmon GP, Apthorp L. Lumbar Spinal Stenosis. In: Skyrme AD, Selmon GP, and Apthorp L, editors. Common Spinal Disorders explained. Remedica 2005; (7):478.

3.AN HS, Anderson PA, Haughton VM. Introduction Disc degeneration: summary. Spine 2004; 29:2677-8. 
4.Kathryn K, Jacob M. Buchowski. Evaluation of Thoracic and Lumbar Radicular Pathology. Who Is a Surgical Candidate and Who Isn't. In: Bridwell KH, Dewald RL, editors. The Textbook of Spinal Surgery. $3^{\text {rd }}$ ed. Philadelphia, Lippincott Williams \& Wilkins 2011; 1(39): 369.

5.Atlas SJ, keller RB, Chang Y, Deyo RA, Singer DE. surgical and non surgical management of sciatica secondary to lumbar disc herniation: five years outcome from the main lumbar spine study. Spine 2001; 26:1179-87.

6.Postacchini F. Lumbar disc herniation: A new equilibrium is needed between nonoperative and operative treatement Spine 2001; 26(6):648-52.

7.Truuumees E. Discogenic Low Back Pain. In: Devlin VJ, editor. Spine Secrets Plus. $2^{\text {nd }}$ ed. Maryland Heights, El Sevier Mosby 2011; 50:340-1.

8.Christopher R. Good, Timothy R. Kuklo: Transforaminal Lumbar Interbody Fusion/Posterior Lumbar Interbody Fusion. In: Bridwell KH, Dewald RL, editors. The Textbook of Spinal Surgery. $3^{\text {rd }}$ ed. Philadelphia, Lippincott Williams \& Wilkins 2011; 2(47):437.

9.Zhou J, Wang B, Dong J, Li X, Zhou X, Fang T, et al. Arch Orthop Trauma Surg 2011; 131:1239-45.

10. Leventhal MR. Spinal anatomy and surgical approaches. In: Crenshow AH, (ed). Campbell's Operative Orthopaedics. 8th ed. St. Louis, London, Toronto, Philadelphia: CV Mosby Co. 1998; Vol. 5: 3493-516.

11. Gray H. Anatomy, Descriptive and Surgical. Philadelphia, Pennsylvania: Running Press 1974; 34-226.

12. Netter FH, Hansen JT. Atlas of Human anatomy. 3rd ed. New York: Icon Learning Systems; 2003.

13. Cailliet R. Low back pain syndrome. Philadelphia: FA Davis Co 1981, 3-19.
14. Louis R. Topographical anatomy and operative approaches: Lumbar spine. In: Surgery of the spine: Surgical anatomy and operative approaches. 1st ed. Berlin, Heidelberg, New York: Springer-Verlag 1983; 264-313.

15. Snell RS. Clinical anatomy for medical students. 2nd ed. Boston: Little brown Co $1981 ; 791-823$.

16. Bogduk N, Macintosch JE. The applied anatomy of the thoraco-lumbar fascia. Spine 1984; 9: 164-70.

17. Vleeming A, Pool-Goudzwaard AL, Stoeckart R, Van Wingerden JP and Snijders CJ. The posterior layer of the thoraco-lumbar fascia: Its function in load transfer from spine to legs. Spine 1995 ; 20 : 753-8

18. White AA, Panjabi MM. Physical properties and functional biomechanics of the spine. In: Clinical biomechanics of the spine. Philadelphia, Tronto: JB Lippincott Company 1978; 1-60

19. Last RJ. Anatomy: regional and applied. 6th ed. Churchill Livingstone 1978; 458-79.

20. Warwick R, Williams PL. Gray's anatomy. 35th ed. 1973, 240-5.

21. Dickson JH, Harrington PR, Erwin WD. Results of reduction and stabilization of the severely fractured thoracic and lumbar spine. J Bone Joint Surg (Am) 1978; 60A: 799-805.

22. Roy-Camille R, Saillant G, Mazel C. Internal fixation of the lumbar spine with pedicle screw plating. Clin Orthop 1986; 203: 7-17

23. Ebraheim NA, Xu R, Darwich M, Yeasting RA. Anatomic relations between the lumbar pedicle and adjacent neural structures. Spine 1997; 22: 2338-41.

24. Krenz J, Troup JDG. The structure of the pars interarticularis of the lower lumbar vertebrae and its relation to the aetiology of spondylolysis. J Bone Joint Surg (Br) 1973; 55B: 735-41.

25. Vaccaro AR, Kepler CK, Rihn JA, Suzuki H, Ratliff JK, Harrop JS, et al. Anatomical 
relationships of the anterior blood vessels to the lower lumbar intervertebral discs: analysis based on magnetic resonance imaging of patients in the prone position. J Bone Joint Surg Am 2012;94(12):1088-94

26. MaMinn RMH, Hutchings RT. A color atlas of human anatomy. London: Wolfe Medical Publications 1977; 73-87.

27. Goethem JWM, Hauwe L, Parizel PM, SpringerLink . Spinal Imaging Diagnostic Imaging of the Spine and Spinal Cord. Berlin, Heidelberg: Springer-Verlag Berlin Heidelberg; 2007.

28. Xu R, Ebraheim NA, Douglas K, Yeasting RA. The projection of the lateral sacral mass on the outer table of the posterior ilium. Spine 1996; 21: 790-4.

29. Hedman TP, Fernie GR. Mechanical response of the lumbar spine to postural loads. Spine 1997; 22: 734-43. 